•生物编目・

\title{
利用红外相机调查西藏洛隆县鸟类和 兽类的物种多样性
}

\author{
郭克疾 ${ }^{1}$ 陆鹏飞 ${ }^{1}$ 石胜超 ${ }^{3}$ 唐梓钧 ${ }^{1}$ 张 同 $^{1}$ 熊嘉武 ${ }^{1}$ 李炳章 ${ }^{2}$ 邓学建 $3^{*}$ \\ 1 (国家林业局中南林业调查规划设计院, 长沙 410014) \\ 2 (西藏自治区林业调查规划研究院, 拉萨 850000) \\ 3 (湖南师范大学生命科学学院, 长沙 410081)
}

\section{Camera trapping survey of mammals and birds in Luolong, Tibet}

\author{
Keji Guo ${ }^{1}$, Pengfei $\mathrm{Lu}^{1}$, Shengchao Shi ${ }^{3}$, Zijun Tang ${ }^{1}$, Tong Zhang ${ }^{1}$, Jiawu Xiong ${ }^{1}$, Bingzhang $\mathrm{Li}^{2}$, Xuejian \\ Deng $^{3 *}$ \\ 1 Central South Forest Inventory and Planning Institute of State Forestry Administration, Changsha 410014 \\ 2 Institute of Forest Inventory, Planning and Research of Tibet Autonomous Region, Lhasa 850000 \\ 3 College of Life Sciences, Hunan Normal University, Changsha 410081
}

红外相机技术作为一种非损伤性的物种调查 和记录技术, 于 20 世纪90年代起开始广泛应用于野 生动物研究(Karanth, 1995; O'Connell et al, 2011; Niedballa et al, 2015)。相对于传统的粪堆法(马建章 等, 1989)、样线法(Stephens et al, 2006)、样带法 (Harris et al, 1992)、大样方调查法(张常智和张明海, 2011)等监测方法，红外相机技术具有非损伤性、可 日夜连续监测的优点, 已成为调查物种多样性、估 算动物种群密度、研究栖息地选择以及记录动物行 为模式的常用手段 (肖治术等, 2014; 张履冰等, 2014)。红外相机技术不仅可以结合个体识别技术和 “捕获一重捕获”模型、最大熵模型和占据模型等应用 于大型猫科动物的种群数量、密度、分布和栖息地 研究(O'Connell et al, 2011; Gopalaswamy et al, 2012; Athreya et al, 2013; Goldberg et al, 2015), 而且结合 近年来发展的随机相遇模型，还能较好地应用于一 些不易进行个体识别的物种(例如有蹄类、啮齿类) 的种群数量和分布研究(Rowcliffe et al, 2008; 章书 声等, 2012)。近年来, 红外相机技术在我国得到了 较好的应用和发展(Pei，1995; 马世来和里查德·何 里来, 1996; Li et al, 2010), 该技术已成功应用到野
生动物生态学研究(章书声等, 2012; 肖文宏等, 2014; Jiang et al, 2015; Qi et al, 2015)、动物行为学研 究(赵玉泽等, 2013; 李峰和蒋志刚, 2014)、稀有物 种的探测与记录(Feng et al, 2001; Sanderson \& Trolle, 2005; 马鸣等, 2006; 肖文宏, 2014)、动物本 底资源调查(宋大昭等, 2014; 肖文宏等, 2014)、生 物多样性监测(刘芳等, 2012; 张明霞等, 2014)及保 护地管理与保护评价(Li et al, 2012)等领域。

2014年5-10月我们在开展西藏自治区第二次 陆生野生动物资源调查的工作中, 通过架设红外相 机对西藏洛隆县的鸟类和兽类进行了调查研究, 以 期能补充完善当地生物资源的基础信息, 为进一步 开展动物区系、珍稀濒危物种种群生态学等方面的 深入系统研究奠定基础, 并为该地区制定保护计划 提供可靠的依据。

\section{1 研究地概况}

研究地位于西藏自治区昌都市洛隆县玉西乡 境内, 地处怒江上游, 横断山脉北段边缘, 西部毗 邻念青唐古拉山脉东南端, 地理坐标为 $30^{\circ} 10^{\prime}-$ $31^{\circ} 50^{\prime} \mathrm{N}, 95^{\circ} 10^{\prime}-95^{\circ} 50^{\prime} \mathrm{E}$ (图1)。该区域在动物地理

收稿日期: 2016-05-11; 接受日期: 2016-08-28

基金项目: 西藏自治区第二次陆生野生动物资源调查项目和国家林业局专项资金项目

* 通讯作者 Author for correspondence. E-mail: dxj8871922@163.com 


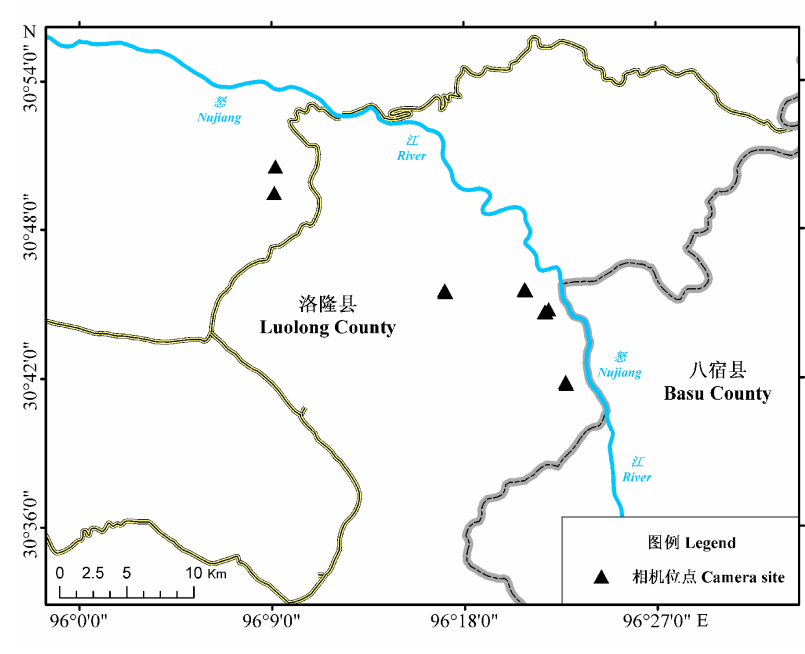

图1 洛隆县研究区域相机位点分布图

Fig.1 The camera sites of the study area in Luolong County

区划上被列为东洋界西南区西南山地亚区, 且为古 北界(青藏区)和东洋界(西南区)的交界区域, 是南 北动物交流的通道, 动物物种丰富, 且分布呈明显 的垂直变化(张荣祖, 2011)。调查区域属藏东南高原 温带半干旱季风气候, 年平均气温 $5.5^{\circ} \mathrm{C}$, 极端最高 气温 $30.6^{\circ} \mathrm{C}$, 极端最低气温 $-22.1^{\circ} \mathrm{C}$ 。年平均降水量 $423.7 \mathrm{~mm}$, 降雨主要集中在5-9月。调查区域海拔为 4,130-4,490 m, 植被类型有针叶林、灌从和草甸。 针叶林主要是云杉(Picea likiangensis)、冷杉(Abies georgei) 林, 灌从主要由锦鸡儿(Caragana spp.)、小 檗(Berberis spp.)、杜鹃(Rhododendron spp.)、绣线 菊 (Spiraea spp.) 等物种组成, 草甸以薹草 (Carex spp.) 草甸、嵩草(Koresia spp.)草甸为主。该调查区 域从未开展过专项生物多样性调查。

\section{2 方法}

\section{1 红外相机布设和数据采集}

在西藏自治区第二次陆生野生动物资源调查 中, 以调查地理单元为单位, 按 $\geq 10 \%$ 的抽样强度, 设立 $10 \mathrm{~km} \times 10 \mathrm{~km}$ 规格的样区, 并在样区内按照一 定走向均匀布设8-12条2-5 km长的调查样线。相机 位点沿着调查样线布置, 主要选择人为活动相对较 少的兽道两侧, 共选择了 7 个位点, 位点间至少相 隔300 m。每个位点布设相邻的相机2台, 相机间隔 10-100 m。相机(夜鹰SG007、DTC560K)用铁丝或 尼龙绳捆绑在合适的树干上, 固定高度为 $0.5 \mathrm{~m}$ 左 右。设置拍摄模式(照片或视频)、连拍(3张)、时间
间隔 $(1 \mathrm{~min})$ 、灵敏度(低)等参数。记录每个相机位 点的GPS信息(经度、纬度以及海拔)。2014年5月28 日至6月8日布设, 2014年10月13日前全部收回。

\section{2 数据处理与统计分析}

首先将照片和视频进行初步整理, 删除没有任 何动物或人为活动的空拍照片和视频。然后鉴定有 效照片和视频中出现的野生动物物种信息(约翰·马 敬能和卡伦·菲利普斯, 2003; 盛和林，2005)。分类 系统主要参考《中国鸟类分类与分布名录》 (郑光美, 2011) 以及《中国哺乳动物多样性及地理分布》(蒋 志刚等, 2015)。最后判断独立事件并删除重复照片 和视频。

独立事件判断标准为: (1)相同或不同物种的不 同个体或人、牲畜的连续照片和视频; (2)相同物种 或人、牲畜的连续照片和视频之间时间间隔大于 30 $\min$; (3)相同物种或人、牲畜的不连续照片和视频 (O’Brien et al, 2003)。符合以上任意一条标准即被定 义为一次独立事件。利用ACDsee 9.0软件对独立事 件照片进行标记和整理, 并用Excel 2013对每个照 片和视频拍摄的日期、时间、物种组成及相机位点 等信息进行汇总。

利用Photoshop CS6和ACDsee 9.0软件对拍摄 到的金钱豹 (Panthera pardus) 照片进行个体识别, 主要识别依据为头部、体两侧、尾部、腿部等部位 的特征花纹。

采用拍摄率(photographic rate, PR)作为动物的 相对多度指标, 计算公式为(刘芳等, 2012): 拍摄率 $(\mathrm{PR})=($ 照片数 $\times 100) /$ 相机日。

\section{3 结果}

\section{1 相机捕获结果统计}

14台红外相机总捕获天数(实际拍摄工作日)共 计 $552 \mathrm{~d}$, 每台相机捕获天数为 $29-136 \mathrm{~d}$, 平均捕获 天数为 $78.9 \mathrm{~d}$ 。拍摄的独立事件总数为 849 次, 其中 $31.3 \%$ 为鸟类, $60.6 \%$ 为兽类, $8.1 \%$ 为人类活动。在所 有拍摄的独立事件中, 无法鉴别的独立事件为 15 次, 仅占总数的 $1.8 \%$; 对其他照片和影像进行物种 鉴定，共发现物种 27 种，隶属于 6 目 13 科。其中，拍 摄到鸟类照片共计 266 张, 其中 6 张不可鉴别物种, 其他照片鉴定出物种 3 目 7 科 15 种, 占总种数的 $55.6 \%$; 兽类照片共计 514 张，其中9张不可鉴别物 种, 其他照片鉴定出物种 3 目 6 科 12 种, 占总种数的 
$44.4 \%$ (附录 1 )。另外, 根据金钱豹个体身上的独特 斑纹进行个体识别, 结果表明共拍摄到至少 4 只金 钱豹。

\section{2 相对丰富度指数}

调查区域中, 灰尾兔(Lepus oiostolus)的拍摄率 (PR 值) 最高 (54.35), 其次为喜马拉雅鹿 (Moschus chrysogaster, 21.92)和血雉(Ithaginis cruentus, 20.65)。鸟类中, PR值相对高的都是鸡形目鸟类, 平 均为 7.61 。而树栖的雀形目则相对较低, 平均为 0.99 。大型猫科动物中, 金钱豹的PR值(1.63)高于雪 豹(Panthera uncial, 0.54)和猞猁(Lynx lynx, 1.27), 且 均高于同为肉食动物的狼(Canis lupus, 0.54); 小型 肉食动物中, 石貂(Martes foina) 的PR值(2.54)高于 豹猫(Prionailurus bengalensis, 0.54)。杂食性的猪獾 (Arctonyx collaris, 3.08) 高于赤狐 (Vulpes vulpes, $1.81)$ 。草食动物中灰尾兔 $\mathrm{PR}$ 值最高, 其次为各种有 蹄类。有蹄类中, 喜马拉雅鹿 (21.92)远高于岩羊 (Pseudois nayaur, 2.17) 和中华瓺羚 (Capricornis milneedwardsii, 1.09)(附录1)。

\section{3 珍稀濒危物种}

本次调查发现的珍稀濒危物种非常丰富(附录 1 )。我国特有物种有 6 种, 占此次记录物种总数的 $22.2 \%$; 国家I级重点保护动物 5种, 国家II级重点保 护动物6种; 自治区I级重点保护动物7种, 自治区 II 级重点保护动物 8 种; 另外被《IUCN濒危物种红色 名录(2016年)》列为“濒危”和“近危”等濒危等级的 物种有 8 种; 被列入《濒危野生动植物种国际贸易公 约》附录的共有 13 种; 被《中国生物多样性红色名 录》列为极危、濒危、易危、近危等濒危等级的物 种共15种。

\section{4 讨论}

通过红外相机对怒江上游区域西藏洛隆县鸟 类和兽类的调查, 我们初步掌握了栖息在洛隆县的 鸟类和兽类的分布概况。为进一步深入了解该地区 鸟类和兽类的分布格局提供了重要的基础资料。目 前已确认的鸟兽共计 27 种, 隶属于 6 目 13 科。本次调 查发现的珍稀濒危物种非常丰富(附录1), 被列入

《IUCN濒危物种红色名录》、《中国脊椎动物红色 名录》(蒋志刚等, 2016)、CITES附录和《国家重点 保护野生动物名录》的物种共 25 种, 占总种数的 $93.0 \%$ 。
根据个体识别统计结果, 该地金钱豹的种群数 量最少有4只, 且根据红外相机捕获的亚成体照片 以及访问调查结果(附录2), 可以推测该地区有较为 稳定的繁殖种群。值得一提的是, 本研究在调查区 域内同时观测到了雪豹和金钱豹两种大型猫科动 物, 特别是在同一个红外相机监测位点先后多次捕 获到雪豹和金钱豹影像(附录2), 表明该区域存在雪 豹和金钱豹同域分布的现象，这在我国十分罕见。 结合各相机位点的物种捕获结果分析, 区域内雪 豹、金钱豹、猞猁、狼等大中型食肉动物可能存在 复杂的竞争关系。这对于开展大型猫科动物的基础 生态学研究和保护有重要的意义。

实践表明, 在洛隆地区通过红外相机调查动物 的野外分布格局是有效的监测手段, 值得青藏高原 地区进一步推广应用。但由于各种客观条件的限制, 本次调查仅限于洛隆县境内约 $187 \mathrm{~km}^{2}$ 的区域, 且 野外调查时间和相机实际工作时间较短, 尚难以全 面准确地评估该地区乌类和兽类的多样性格局以 及重要物种的分布和种群密度, 特别是金钱豹、雪 豹等关键物种的分布和密度。为了进一步掌握野生 动物的资源现状, 亟需在更大范围内开展长期调查 与监测工作, 合理增加红外相机的数量, 扩大监测 区域和延长监测时间, 从而更全面地掌握以洛隆县 为代表的藏东南地区野生动物多样性及其分布 格局。

致谢: 本文调查工作得到了西藏自治区林业厅、昌 都市林业局、洛隆县林业局等各级林业主管部门的 支持和协助，特此感谢。

\section{参考文献}

Athreya V, Odden M, Linnell JDC, Krishnaswamy J, Karanth KU (2013) Big cats in our backyards: persistence of large carnivores in a human dominated landscape in India. PLoS ONE, 8, e57872.

Feng L, Wang T, Mou P, Kou X, Ge J (2001) First image of an Amur leopard recorded in China. Cat News, 55, 9.

Goldberg JF, Tempa T, Norbu N, Hebblewhite M, Mills LS, Wangchuk TR, Lukacs P (2015) Examining temporal sample scale and model choice with spatial capture-recapture models in the common leopard Panthera pardus. PLoS ONE, 10, e0140757.

Gopalaswamy AM, Royle JA, Delampady M, Nichols JD, Karanth KU, Macdonald DW (2012) Density estimation in tiger populations: combining information for strong inference. Ecology, 93, 1741-1751. 
Harris RB, Liu YS, Cai GQ, Bart WO (1992) Line transects for estimating density of musk deer pellet groups. Acta Theriologica Sinica, 12, 302-305. (in Chinese) [Harris RB, 刘永生, 蔡桂全, Bart WO (1992) 使用样线法进行马榭粪 堆密度的测量. 兽类学报, 12, 302-305.]

Jiang G, Qi J, Wang G, Shi Q, Darman Y, Hebblewhite M, Miquelle DG, Li Z, Zhang X, Gu J, Chang Y, Zhang M, Ma $J$ (2015) New hope for the survival of the Amur leopard in China. Scientific Reports, 5, 15475.

Jiang ZG, Ma Y, Wu Y, Wang YX, Zhou KY, Liu SY, Feng ZJ (2015) China's Mammal Diversity and Geographic Distribution. Science Press, Beijing. (in Chinese) [蒋志刚, 马勇, 吴毅, 王应祥, 周开亚, 刘少英, 冯祚建 (2015) 中国哺 乳动物多样性及地理分布. 科学出版社, 北京.]

Jiang ZG, Jiang JP, Wang YZ, Zhang E, Zhang YY, Li LL, Xie F, Cai B, Cao L, Zheng GM, Dong L, Zhang ZW, Ding P, Luo ZH, Ding CQ, Ma ZJ, Tang SH, Cao WX, Li CW, Hu HJ, Ma Y, Wu Y, Wang YX, Zhou KY, Liu SY, Chen YY, Li JT, Feng ZJ, Wang Y, Wang B, Li C, Song XL, Cai L, Zang CX, Zeng Y, Meng ZB, Fang HX, Ping XG (2016) Red List of China's Vertebrates. Biodiversity Science, 24, 500-551. (in Chinese with English abstract) [蒋志刚, 江建 平，王跃招，张鹗，张雁云，李立立，谢锋，蔡波，曹亮， 郑光美, 董路, 张正旺, 丁平, 罗振华, 丁长青, 马志军, 汤宋华, 曹文宣, 李春旺, 胡慧建, 马勇, 吴毅, 王应祥, 周开亚, 刘少英, 陈跃英, 李家堂, 冯祚建, 王燕, 王斌, 李成, 宋雪琳, 蔡蕾, 藏春金金, 曾岩, 孟智斌, 方红霞, 平 晓鸽 (2016) 中国脊椎动物红色名录. 生物多样性, 24, 500-551.]

Karanth KU (1995) Estimating tiger Panthera tigris populations from camera-trap data using capture-recapture models. Biological Conservation, 71, 333-338.

Li F, Jiang ZG (2014) Is nocturnal rhythm of Asian badger (Meles leucurus) caused by human activity? A case study in the eastern area of Qinghai Lake. Biodiversity Science, 22, 758-763. (in Chinese with English abstract) [李峰, 蒋志刚 (2014) 狗獾夜间活动节律是受人类活动影响而形成的 吗? 基于青海湖地区的研究实例. 生物多样性, 22, 758763.]

Li S, Wang D, Gu X, Mcshea WJ (2010) Beyond pandas, the need for a standardized monitoring protocol for large mammals in Chinese nature reserves. Biodiversity and Conservation, 19, 3195-3206.

Li S, Mcshea WJ, Wang D, Lu Z, Gu X (2012) Gauging the impact of management expertise on the distribution of large mammals across protected areas. Diversity and Distributions, 18, 1166-1176.

Liu F, Li DQ, Wu JG (2012) Using infra-red cameras to survey wildlife in Beijing Songshan National Nature Reserve. Acta Ecologica Sinica, 32, 730-739. (in Chinese with English abstract) [刘芳, 李迪强, 吴记贵 (2012) 利用红外相机调查 北京松山国家级自然保护区的野生动物物种. 生态学报, 32, 730-739.]
Ma JZ, Chang H, Meng XL (1989) A study on the frequency distribution of red deer's pellet groups and its practical application. Chinese Wildlife, (6), 6-12. (in Chinese with English abstract) [马建章, 常弘, 孟宪林 (1989) 马鹿粪堆分 布型的研究及其应用. 野生动物学报, (6), 6-12.]

Ma M, Xu F, Chundawat RS, Jumabay K, Wu YQ, Aizezi, Zhu MH (2006) Camera trapping of snow leopards for the photo capture rate and population size in the Muzat Valley of Tianshan Mountains. Acta Zoologica Sinica, 52, 788-793. (in Chinese with English abstract) [马鸣, 徐峰, Chundawat RS, Jumabay K, 吴逸群, 艾则孜, 朱玛洪 (2006) 利用自 动照相术获得天山雪豹拍摄率与个体数量. 动物学报, 52, 788-793.]

Ma SL, Harris RB (1996) Use of remote camera systems to document wildlife species presence in forested areas of Yunnan. Zoological Research, 17, 360, 370. (in Chinese) [马世来, 里查德·何里来 (1996) 自动感应照像系统在野 生动物调查中的应用. 动物学研究, 17, 360, 370.]

Mackinnon J, Phillipps K (2003) A Field Guide to the Birds of China. Hunan Education Publishing House, Changsha. (in Chinese) [约翰·马敬能, 卡伦·菲利普斯 (2003) 中国鸟类 野外手册. 湖南教育出版社, 长沙.]

Niedballa J, Sollmann R, Mohamed AB, Bender J, Wilting A (2015) Defining habitat covariates in camera-trap based occupancy studies. Scientific Reports, 5, 17041.

O'Brien TG, Kinnaird MF, Wibisono HT (2003) Crouching tigers, hidden prey: Sumatran tiger and prey populations in a tropical forest landscape. Animal Conservation, 6, 131-139.

O'Connell AF, Nichols JD, Karanth KU (2011) Camera Traps in Animal Ecology: Methods and Analyses. Springer, New York.

Pei K (1995) Activity rhythm of the spinous country rat (Niviventer coxingi) in Taiwan. Zoological Studies, 34(1), $55-58$.

Qi J, Shi Q, Wang G, Li Z, Sun Q, Hua Y, Jiang G (2015) Spatial distribution drivers of Amur leopard density in northeast China. Biological Conservation, 191, 258-265.

Rowcliffe J, Field J, Turvey SC (2008) Estimating animal density using camera traps without the need for individual recognition. Journal of Applied Ecology, 45, 1228-1236.

Sanderson JG, Trolle M (2005) Monitoring elusive mammals: unattended cameras reveal secrets of some of the world's wildest places. American Scientist, 93, 148-155.

Sheng HL (2005) Atlas of Reptilia of China. Henan Science and Technology Press, Zhengzhou. (in Chinese) [盛和林 (2005) 中国哺乳动物图鉴. 河南科学技术出版社, 郑州.]

Song DZ, Wang BP, Jiang JY, Wan SP, Cui SM, Wang TM, Feng LM (2014) Using camera trap to monitor a North Chinese leopard (Panthera pardus japonesis) population and their main ungulate prey. Biodiversity Science, 22, 733-736. (in Chinese with English abstract) [宋大昭, 王卜平, 蒋进 原, 万绍平, 崔士明, 王天明, 冯利民 (2014) 山西晋中 庆城林场华北豹及其主要猎物种群的红外相机监测. 生 
物多样性, 22, 733-736.]

Stephens PA, Zaumyslova OY, Miquelle DG, Myslenkov AI, Hayward GD (2006) Estimating population density from indirect sign: track counts and the Formozov-Malyshev-Pereleshin formula. Animal Conservation, 9, 339-348.

Xiao WH (2014) Amur Tiger (Panthera tigris altaica) and Its Prey in Hunchun Nature Reserve, Jilin, China: Their Population Size, Distribution and Occupancy. PhD dissertation, Beijing Normal University, Beijing. (in Chinese with English abstract) [肖文宏 (2014) 东北虎(Panthera tigris altaica) 与猎物的种群分布、数量和占据研究. 博士学位论 文, 北京师范大学, 北京.]

Xiao WH, Feng LM, Zhao XD, Yang HT, Dou HL, Cheng YC, Mou P, Wang TM, Ge JP (2014) Distribution and abundance of Amur tiger, Amur leopard and their ungulate prey in Hunchun National Nature Reserve, Jilin. Biodiversity Science, 22, 717-724. (in Chinese with English abstract) [肖文 宏, 冯利民, 赵小丹, 杨海涛, 窦海龙, 程艳超, 牟溥, 王 天明, 葛剑平 (2014) 吉林珲春自然保护区东北虎和东 北豹及其有蹄类猎物的多度与分布. 生物多样性, 22 , 717-724.]

Xiao ZS, Li XH, Jiang GS (2014) Applications of camera trapping to wildlife surveys in China. Biodiversity Science, 22, 683-684. (in Chinese) [肖治术, 李欣海, 姜广顺 (2014) 红外相机技术在我国野生动物监测研究中的应用. 生物 多样性, 22, 683-684.]

Zhang CZ, Zhang MH (2011) Population status and dynamic trends of Amur tiger's prey in Eastern Wandashan Mountain, Heilongjiang Province. Acta Ecologica Sinica, 31, 6481-6487. (in Chinese with English abstract) [张常智, 张 明海 (2011) 黑龙江省东完达山地区东北虎猎物种群现 状及动态趋势. 生态学报, 31, 6481-6487.]

Zhang LB, Cui SP, Huang YJ, Chen DQ, Qiao HJ, Li CW, Jiang ZG (2014) Infrared camera traps in wildlife research and monitoring in China: issues and insights. Biodiversity
Science, 22, 696-703. (in Chinese with English abstract) [张 履冰, 崔绍朋, 黄元骏, 陈代强, 乔慧捷, 李春旺, 蒋志 刚 (2014) 红外相机技术在我国野生动物监测中的应用: 问题与限制. 生物多样性, 22, 696-703.]

Zhang MX, Cao L, Quan RC, Xiao ZS, Yang XF, Zhang WF, Wang XZ, Deng XB (2014) Camera trap survey of animals in Xishuangbanna Forest Dynamics Plot, Yunnan. Biodiversity Science, 22, 830-832. (in Chinese) [张明霞, 曹林, 权 锐昌, 肖治术, 杨小飞, 张文富, 王学志, 邓晓保 (2014) 利用红外相机监测西双版纳森林动态样地的野生动物多 样性. 生物多样性, 22, 830-832.]

Zhang RZ (2011) Zoogeography of China. Science Press, Beijing. (in Chinese) [张荣祖 (2011) 中国动物地理. 科学出 版社, 北京.]

Zhang SS, Bao YX, Wang YN, Fang PF, Ye B (2012) Activity rhythms of black muntjac (Muntiacus crinifron) revealed with infrared camera. Acta Theriologica Sinica, 32, 368-372. (in Chinese with English abstract) [章书声, 鲍毅 新, 王艳妮, 方平福, 叶涁 (2012) 基于红外相机技术的 黑鹿活动节律. 兽类学报, 32, 368-372.]

Zhao YZ, Wang ZC, Xu JL, Luo X, An LD (2013) Activity rhythm and behavioral time budgets of wild Reeves's pheasant (Syrmaticus reevesii) using infrared camera. Acta Ecologica Sinica, 33, 6021-6027. (in Chinese with English abstract) [赵玉泽, 王志臣, 徐基良, 罗旭, 安丽丹 (2013) 利用红外照相技术分析野生白冠长尾雉活动节律及时间 分配. 生态学报, 33, 6021-6027.]

Zheng GM (2011) A Checklist on the Classification and Distribution of the Birds of China, 2nd edn. Science Press, Beijing. (in Chinese) [郑光美 (2011) 中国鸟类分类与分布名 录. 科学出版社, 北京.]

(责任编委: 丁平 责任编辑: 间文杰)

\section{附录 Supplementary Material}

附录1 利用红外相机拍摄到的鸟类和兽类名录

Appendix 1 List of birds and mammals captured by camera traps http:// www.biodiversity-science.net/fileup/PDF/2016128-1.pdf

附录2 红外相机所拍摄的兽类和鸟类代表性照片

Appendix 2 Representative mammals and birds recorded from camera traps http:// www.biodiversity-science.net/fileup/PDF/2016128-2.pdf 
郭克疾, 陆鹏飞, 石胜超, 唐梓钧, 张同, 熊嘉武, 李炳章, 邓学建. 利用红外相机调查西藏洛隆县鸟类和兽类的物种多样性. 生物多样性, 2016, 24 (9): 1077-1081. http://www.biodiversity-science.net/CN/10.17520/biods.2016128

附录1 利用红外相机拍摄到的鸟类和兽类名录

Appendix 1 List of birds and mammals captured by camera traps

\begin{tabular}{|c|c|c|c|c|c|c|c|c|c|}
\hline \multirow[b]{2}{*}{ 物种 Species } & \multicolumn{6}{|c|}{ 保护级别 Protection level } & \multirow[b]{2}{*}{$\begin{array}{l}\text { 相机位点 } \\
\text { 计数 } \\
\text { Camera } \\
\text { sites }\end{array}$} & \multirow[b]{2}{*}{$\begin{array}{c}\text { 独立有效照片数 } \\
\text { 量合计 } \\
\text { Independent } \\
\text { photos }\end{array}$} & \multirow[b]{2}{*}{$\begin{array}{c}\text { 拍摄率 } \\
\text { (每百日捕获数) } \\
\text { Capture rate }\end{array}$} \\
\hline & $\begin{array}{c}\text { 国家级 } \\
\text { National } \\
\text { level }\end{array}$ & $\begin{array}{c}\text { 自治区级 } \\
\text { Autonomous } \\
\text { region level }\end{array}$ & IUCN & CITES & $\begin{array}{c}\text { 中国生物多样性红 } \\
\text { 色名录 } \\
\text { Red List of China's } \\
\text { Biodiversity }\end{array}$ & $\begin{array}{l}\text { 特有种 } \\
\text { Endemic } \\
\text { species }\end{array}$ & & & \\
\hline 鸟纲 AVES & & & & & & & & 260 & 38.59 \\
\hline \multicolumn{10}{|l|}{ 鸡形目 GALLIFORMES } \\
\hline \multicolumn{10}{|l|}{ 松鸡科 Tetraonidae } \\
\hline 斑尾榛鸡 Bonasa sewerzowi & I & I & NT & & NT & $\sqrt{ }$ & 1 & 23 & 4.17 \\
\hline \multicolumn{10}{|l|}{ 雉科 Phasianidae } \\
\hline 黄喉雉敦 Tetraophasis szechenyii & I & I & LC & & $\mathrm{VU}$ & $\sqrt{ }$ & 4 & 24 & 4.35 \\
\hline 藏雪鸡 Tetraogallus tibetanus & II & II & LC & I & NT & & 1 & 1 & 0.18 \\
\hline 血雉 Ithaginis cruentus & II & II & LC & II & NT & & 6 & 114 & 20.65 \\
\hline 白马鸡 Crossoptilon crossoptilon & II & II & NT & I & NT & $\sqrt{ }$ & 6 & 48 & 8.70 \\
\hline \multicolumn{10}{|l|}{ 鴷形目 PICIFORMES } \\
\hline \multicolumn{10}{|l|}{ 啄木鸟科 Picidae } \\
\hline 灰头绿豚木鸟 Picus canus & 三有 & & LC & & LC & & 1 & 1 & 0.18 \\
\hline \multicolumn{10}{|l|}{ 雀形目 PASSERIFORMES } \\
\hline \multicolumn{10}{|l|}{ 鸦科 Corvidae } \\
\hline 大嘴乌鸦 Corvus macrorhynchos & 三有 & & LC & & LC & & 3 & 8 & 1.45 \\
\hline 渡鸦 Corvus corax & 三有 & & LC & & LC & & 1 & 1 & 0.18 \\
\hline \multicolumn{10}{|l|}{ 冻科 Turdidae } \\
\hline 白喉红尾鸲 Phoenicurus schisticeps & & & LC & & LC & & 1 & 6 & 1.09 \\
\hline 长尾地鸫 Zoothera dixoni & & & LC & & LC & & 1 & 3 & 0.54 \\
\hline 棕背黑头鸫 Turdus kessleri & 三有 & & LC & & LC & & 3 & 7 & 1.27 \\
\hline \multicolumn{10}{|l|}{ 画眉科 Timaliidae } \\
\hline 大噪鸲 Garrulax maximus & 三有 & & LC & & LC & $\sqrt{ }$ & 4 & 6 & 1.09 \\
\hline 灰腹噪鹋 Garrulax henrici & 三有 & & LC & & LC & $\sqrt{ }$ & 3 & 14 & 2.54 \\
\hline 棕草鸲 Babax koslowi & 三有 & & NT & & & $\checkmark$ & 1 & 3 & 0.54 \\
\hline \multicolumn{10}{|l|}{ 燕雀科 Fringillidae } \\
\hline 白眉朱雀 Carpodacus thura & 三有 & & LC & & & & 1 & 1 & 0.18 \\
\hline 兽纲 MAMMALIA & & & & & & & & 505 & 91.48 \\
\hline 食肉目 CARNIVORA & & & & & & & & & \\
\hline
\end{tabular}


郭克疾, 陆鹏飞, 石胜超, 唐梓钧, 张同, 熊嘉武, 李炳章, 邓学建. 利用红外相机调查西藏洛隆县鸟类和兽类的物种多样性. 生物多样性, 2016, 24 (9): 1077-1081. http://www.biodiversity-science.net/CN/10.17520/biods.2016128

\begin{tabular}{|c|c|c|c|c|c|c|c|c|c|}
\hline \multirow[b]{2}{*}{ 物种 Species } & \multicolumn{6}{|c|}{ 保护级别 Protection level } & \multirow{2}{*}{$\begin{array}{l}\text { 相机位点 } \\
\text { 计数 } \\
\text { Camera } \\
\text { sites }\end{array}$} & \multirow{2}{*}{$\begin{array}{c}\text { 独立有效照片数 } \\
\text { 量合计 } \\
\text { Independent } \\
\text { photos }\end{array}$} & \multirow[b]{2}{*}{$\begin{array}{c}\text { 拍摄率 } \\
\text { (每百日捕获数) } \\
\text { Capture rate }\end{array}$} \\
\hline & $\begin{array}{c}\text { 国家级 } \\
\text { National } \\
\text { level }\end{array}$ & $\begin{array}{c}\text { 自治区级 } \\
\text { Autonomous } \\
\text { region level }\end{array}$ & IUCN & CITES & $\begin{array}{c}\text { 中国生物多样性红 } \\
\text { 色名录 } \\
\text { Red List of China's } \\
\text { Biodiversity } \\
\end{array}$ & $\begin{array}{l}\text { 特有种 } \\
\text { Endemic } \\
\text { species }\end{array}$ & & & \\
\hline \multicolumn{10}{|l|}{ 犬科 Carnidae } \\
\hline 狼 Canis lupus & 三有 & I & LC & II & NT & & 2 & 3 & 0.54 \\
\hline 赤狐 Vulpes vulpes & 三有 & I & LC & III & NT & & 2 & 10 & 1.81 \\
\hline \multicolumn{10}{|l|}{ 鼠科 Mustelidae } \\
\hline 石貂 Martes foina & II & II & LC & III & EN & & 1 & 14 & 2.54 \\
\hline 猪獾 Arctonyx collaris & 三有 & & NT & 未列入 & NT & & 6 & 17 & 3.08 \\
\hline \multicolumn{10}{|l|}{ 猫科 Felidae } \\
\hline 豹猫 Prionailurus bengalensis & 三有 & II & LC & I & VU & & 2 & 3 & 0.54 \\
\hline 猞猁 Lynx lynx & II & II & LC & II & EN & & 2 & 7 & 1.27 \\
\hline 金钱豹 Panthera pardus & I & I & NT & I & EN & & 4 & 9 & 1.63 \\
\hline 雪豹 Panthera uncia & I & I & EN & I & EN & & 2 & 3 & 0.54 \\
\hline \multicolumn{10}{|l|}{ 鲸偶蹄目 CETARTIODACTYLA } \\
\hline \multicolumn{10}{|l|}{ 桹科 Moschidae } \\
\hline $\begin{array}{l}\text { 喜马拉雅磨 (白腹㯍) Moschus } \\
\text { chrysogaster }\end{array}$ & I & I & EN & II & EN & & 5 & 121 & 21.92 \\
\hline \multicolumn{10}{|l|}{ 牛科 Bovidae } \\
\hline 岩羊 Pseudois nayaur & II & II & LC & III & LC & & 4 & 12 & 2.17 \\
\hline 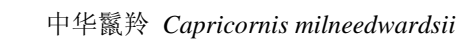 & II & II & NT & I & $\mathrm{VU}$ & & 2 & 6 & 1.09 \\
\hline \multicolumn{10}{|l|}{ 兔形目 LAGOMORPHA } \\
\hline \multicolumn{10}{|l|}{ 兔科 Leporidae } \\
\hline 灰尾兔 Lepus oiostolus & 三有 & & LC & 未列入 & LC & & 5 & 300 & 54.35 \\
\hline
\end{tabular}


郭克疾，陆鹏飞，石胜超，唐梓钧，张同，熊嘉武，李炳章，邓学建. 利用红外相机调查西藏洛隆县鸟类和兽类的物种 多样性. 生物多样性, 2016, 24 (9): 1077-1081.

http://www.biodiversity-science.net/CN/10.17520/biods.2016128

附录 2 红外相机所拍摄的兽类和鸟类代表性照片

Appendix 2 Representative mammals and birds recorded from camera traps

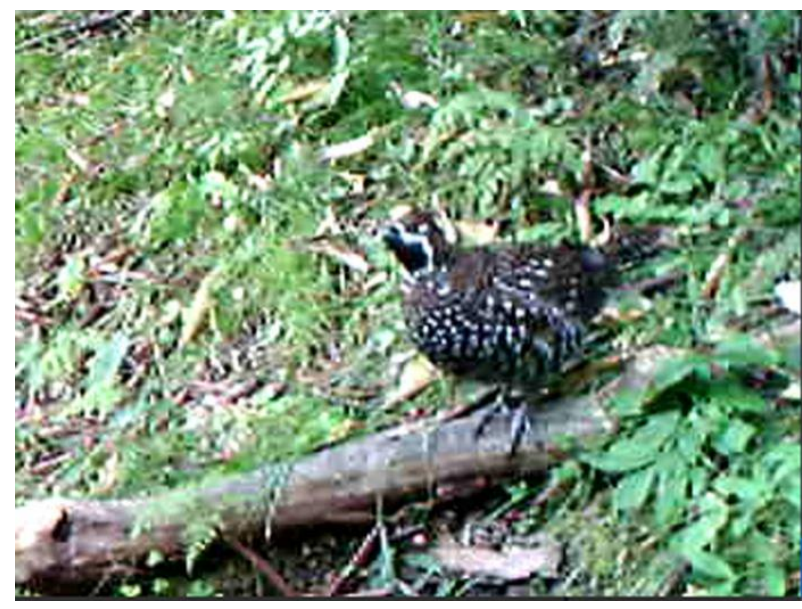

A 斑尾榛鸡 Bonasa sewerzowi

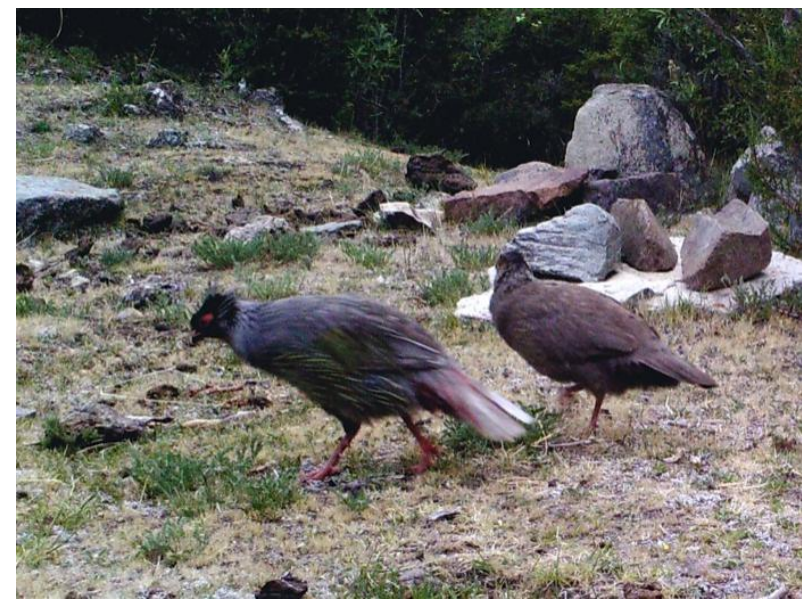

C 血雉 Ithaginis cruentus

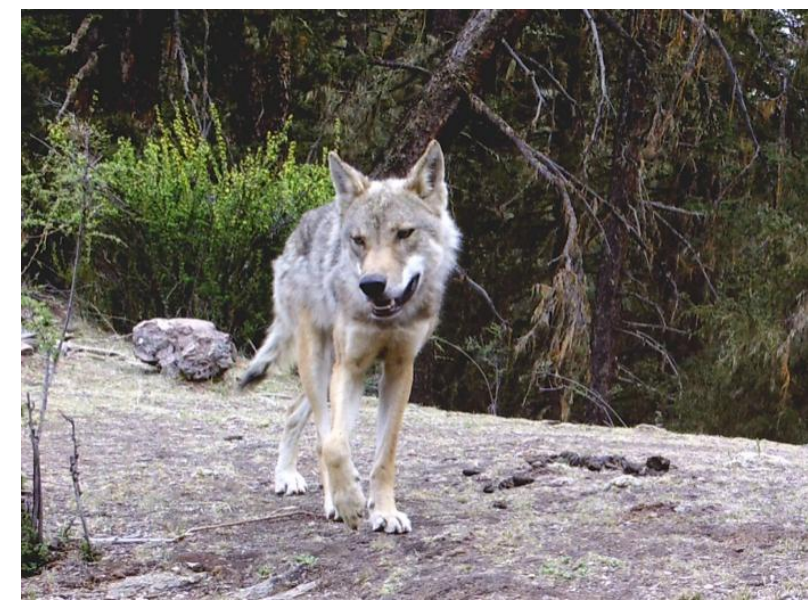

E 狼 Canis lupus

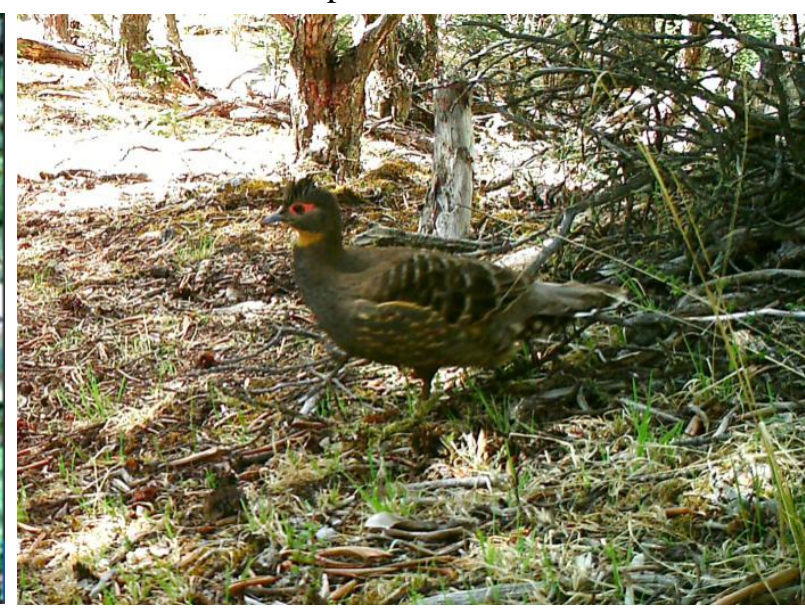

B 黄喉雉敦 Tetraophasis szechenyii

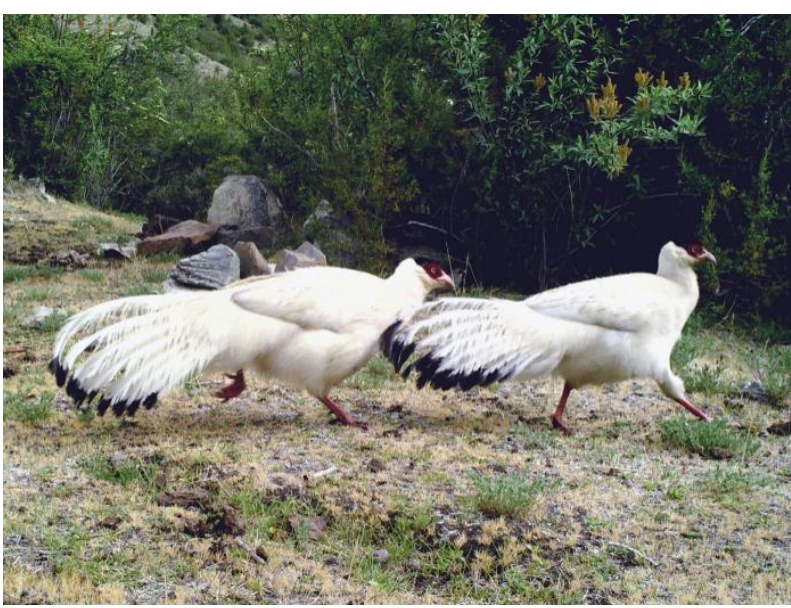

D 白马鸡 Crossoptilon crossoptilon

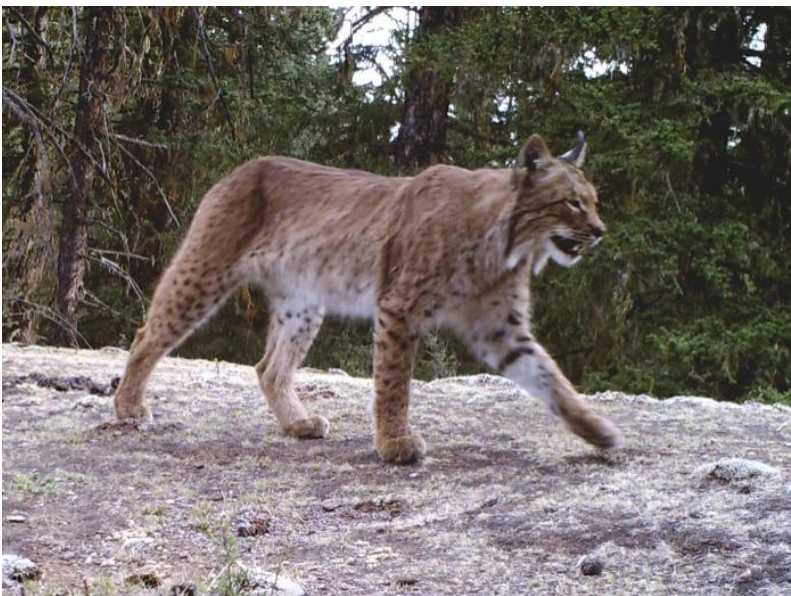

F 猞猁 Lynx lynx 
郭克疾，陆鹏飞，石胜超，唐梓钧，张同，熊嘉武，李炳章，邓学建. 利用红外相机调查西藏洛隆县鸟类和兽类的物种 多样性. 生物多样性, 2016, 24 (9): 1077-1081.

http://www.biodiversity-science.net/CN/10.17520/biods.2016128

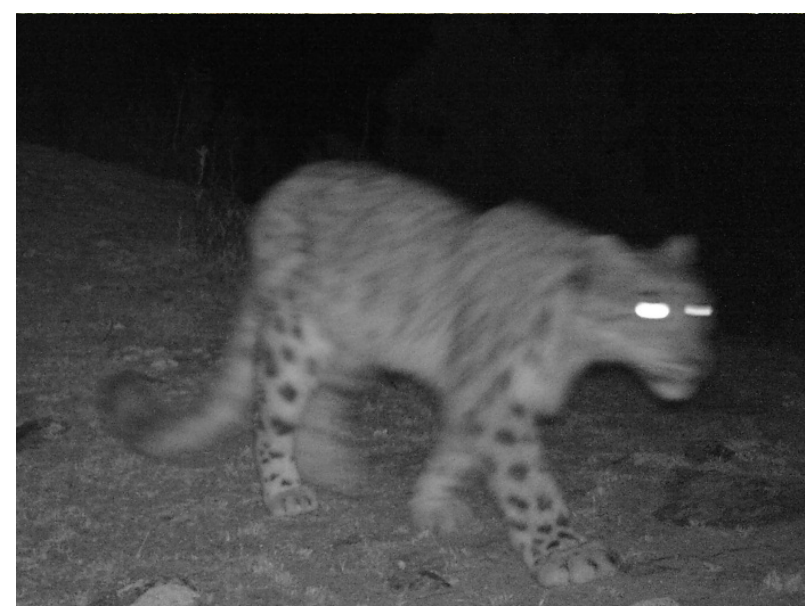

G 雪豹 Panthera uncia

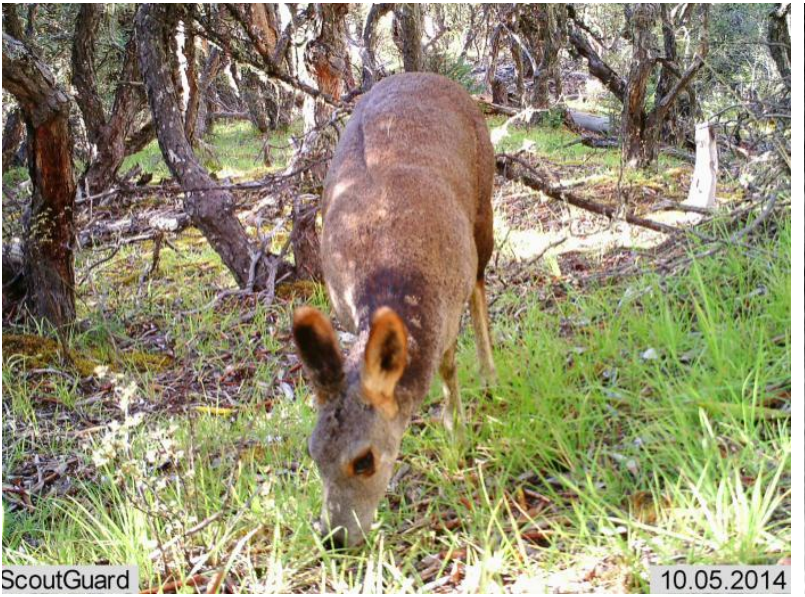

I 喜马拉雅麝 Moschus chrysogaster

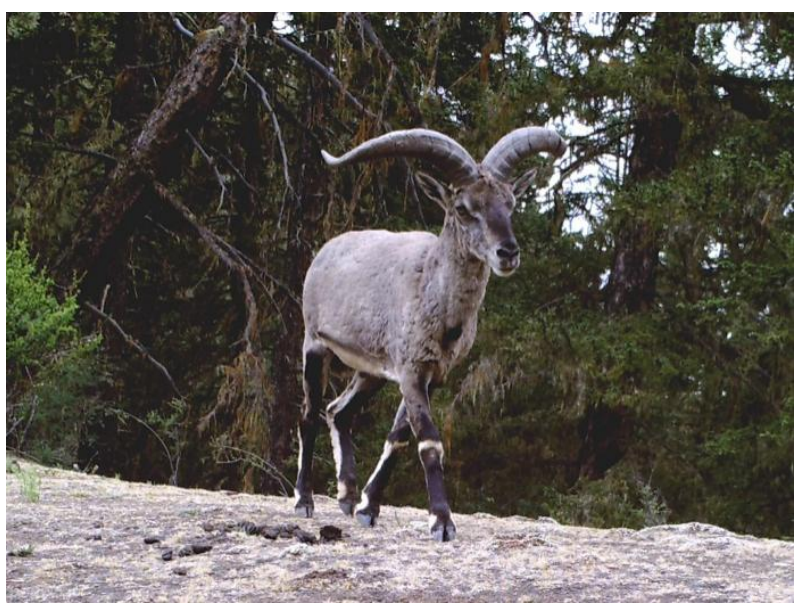

K 岩羊 Pseudois nayaur

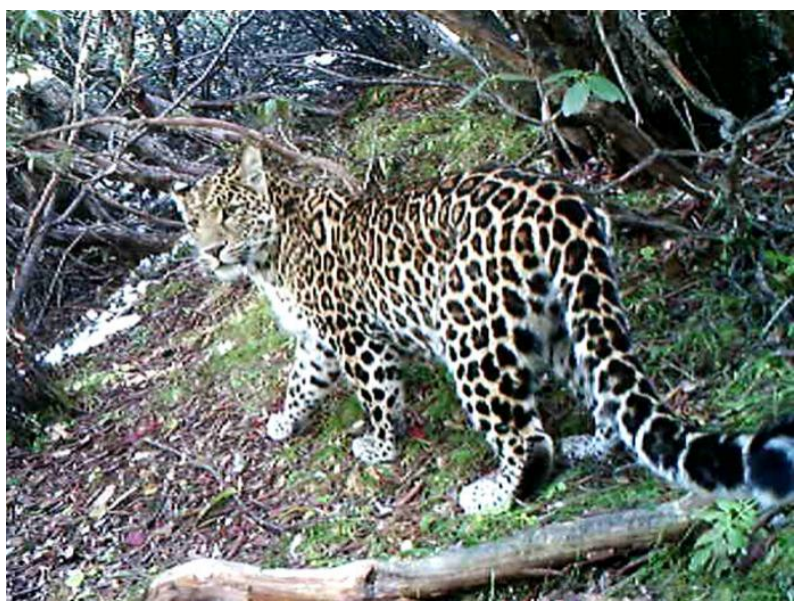

$\mathrm{H}$ 金钱豹 Panthera pardus（亚成体）

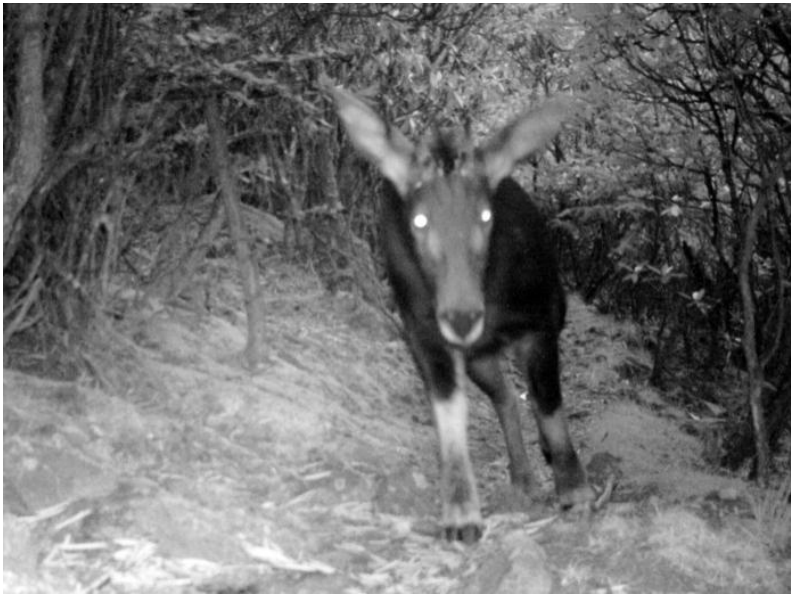

$\mathrm{J}$ 中华瓺羚 Capricornis milneedwardsii

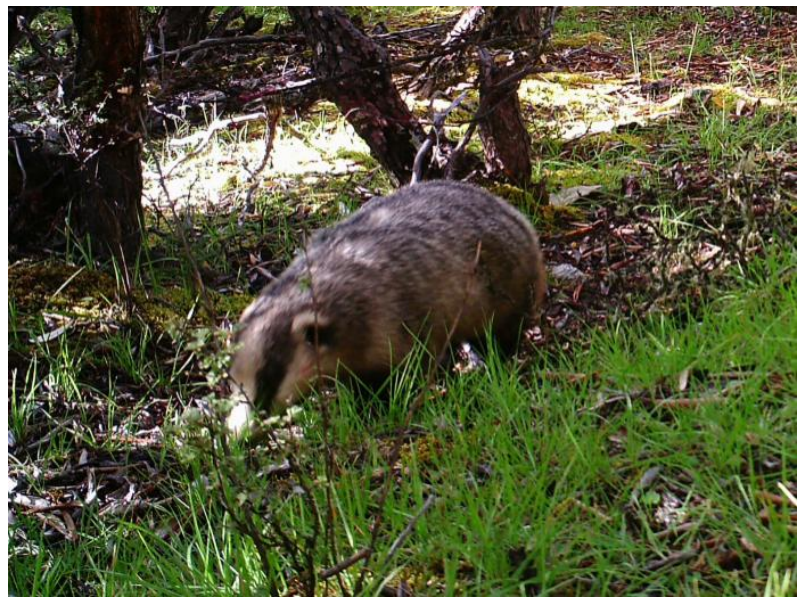

L 猪獾 Arctonyx collaris 